\title{
Quaderni
}

QUADERNI Communication, technologies, pouvoir

89 | Hiver 2015-2016

Penser l'avenir : le CESTA, un think tank atypique

\section{Le Grand Paris qui mange : un projet politique et culturel en émergence?}

Première partie. La transition alimentaire métropolitaine : quels enjeux, quelles formes et quelles invitations citoyennes?

Dominique Pagès

\section{(2) OpenEdition}

Journals

Édition électronique

URL : http://journals.openedition.org/quaderni/960

DOI : 10.4000 /quaderni.960

ISSN : 2105-2956

Éditeur

Les éditions de la Maison des sciences de l'Homme

Édition imprimée

Date de publication : 5 janvier 2016

Pagination : 75-85

Référence électronique

Dominique Pagès, "Le Grand Paris qui mange : un projet politique et culturel en émergence ? », Quaderni [En ligne], 89 | Hiver 2015-2016, mis en ligne le 05 janvier 2018, consulté le 30 avril 2019 URL : http://journals.openedition.org/quaderni/960; DOI : 10.4000/quaderni.960 


\section{Politique}

\section{Le Grand Paris}

qui mange : un

projet politique

et culturel en

émergence?

Première partie. La

transition alimentaire

métropolitaine : quels enjeux, quelles formes et quelles invitations

citoyennes?

\section{Dominique Pagès}

Celsa, GRIPIC Université Paris Sorbonne
Notre alimentation (et plus particulièrement le régime alimentaire des pays occidentaux) contribue de façon significative aux émissions de gaz à effets de serre (notamment à travers la surconsommation de protéines animales et de produits transformés mais aussi à travers le transport). Les prévisions de croissance urbaine à l'horizon 2050 amènent à tirer la sonnette d'alarme sur les questions charnières d'approvisionnement, de sécurité mais aussi de souveraineté alimentaires des villes.

Le projet du Grand Paris semble une belle occasion pour questionner les mutations environnementales, sociales, culturelles et symboliques de nos systèmes alimentaires métropolitains et pour envisager une réponse globale et concertée aux questions de transition alimentaire. Penser en termes politiques « le Grand Paris qui mange », c'est souhaiter que la métropole élabore une diversité croissante de compétences spécifiques pour agir sur l'alimentation qui relève encore principalement des politiques nationales et européennes. C'est avoir l'ambition de faire du modèle alimentaire du Grand Paris où se déploie déjà une diversité de projets agri-urbains un exemple tant au niveau national qu'international.

Ce premier article se propose de revenir sur les enjeux et déterminants des villes nourricières et notamment leur approche de l'agriculture urbaine, sur les formes urbaines qu'elles initient. Le second article à paraître dans un prochain numéro de Quaderni portera quant à lui sur les processus culturels et patrimoniaux que ces villes déploient pour dépasser une approche simplement fonctionnelle et environnementale et ainsi s'ouvrir plus avant à des appropriations citoyennes, 
économiques et artistiques renouvelées ${ }^{1}$.

\section{Les villes nourricières : enjeux, valeurs et gouvernance}

L'actualité récente nous dit combien la question des métropoles en transition alimentaire devient centrale dans les débats sur la ville durable: l'Exposition Universelle de Milan qui a récemment fermé ses portes a posé la question de la nécessaire évolution des systèmes alimentaires en proposant à ses exposants de réfléchir à la thématique « Nourrir la planète, énergie pour la vie ». Plus proche géographiquement de nous, la déclaration de Rennes de juillet 2014, en faveur des systèmes alimentaires territorialisés, lancée à l'occasion d'un colloque organisé par l'Assemblée des Régions de France et la Région Bretagne, mais aussi les diverses initiatives de la Mairie de Paris concernant le plan alimentation dans les lieux publics (écoles, crèches, maisons de retraite, hôpitaux, etc. ${ }^{2}$ ), ses initiatives en termes d'agriculture urbaine et de végétalisation des toits, le lancement du permis de végétaliser ont confirmé cette volonté de consolider voire de construire des politiques publiques alimentaires urbaines à part entière, à repenser non seulement leurs conceptions de la nature et de la production alimentaire mais aussi leur organisation ${ }^{3}$.

\section{Penser des réponses urbaines à nos crises alimentaires}

Ces mutations donnent lieu à une diversité de publications dont certaines, francophones, installent progressivement dans notre horizon intellectuel un modèle : celui de la ville durable et plus avant celui de la ville fertile ${ }^{4}$, de la ville comestible, nourricière ${ }^{5}$ ou vivrière, celui de la ville dite du vivant, autant de désignations qui témoignent d'une prise en compte encore hésitante des questions alimentaires et, peut-être, d'un discours relevant plus de la promesse que de la réalisation achevée, du récit de refondation que de politiques publiques globales pleinement abouties et assumées.

La préoccupation de ces ouvrages porte sur la crise annoncée de nos systèmes alimentaires : en 2050 nous serons 9 milliards et $70 \%$ des individus habiteront les villes. Or le modèle industriel alimentaire actuel, qui est énergivore, polluant et dangereux tant pour la santé que pour le climat, aura sans doute épuisé les sols, aggravé l'empreinte écologique des territoires s'il n'est pas remis en question. L'étalement urbain aura raréfié les espaces agricoles.

Ainsi, plus les sociétés se concentrent dans la ville, plus elles doivent veiller à leur approvisionnement, mieux organiser les transports, repenser tant la production que la maîtrise foncière des sols agricoles. La métropole qui mange, c'est à la fois celle qui « mange de la nourriture et celle qui mange le territoire nécessaire pour produire celle-ci».

Progressivement, depuis 20 ans, s'est installée l'image de la ville fertile, celle d'une cité harmonieuse et pacifiée, salvatrice et résiliente, réconciliée avec la nature. Aux imageries de l'abondance voire de l'excès des villes nourricières du passé ont succédé celles de la frugalité, de la viabilité et de la sobriété. La ville nourricière, cette utopie bien ancienne qui depuis l'Antiquité se métamorphose et pose la question du rapport de 
l'humanité urbaine au monde animal et végétal, semble donc vouloir de plus en plus occuper l'agenda des politiques publiques.

Plus récemment et plus concrètement, un certain nombre d'initiatives locales et internationales ont émergé pour accompagner la mise en œuvre de stratégies alimentaires susceptibles de contribuer au développement durable. Des réseaux internationaux (comme l'International network of ressource center on urban agriculture and food security - http://www.ruaf.org/), des organisations gouvernementales (comme le Growing power, un organisme national américain à but non lucratif, un projet national de recherche et d'expérimentations - http://www. growingpower.org/) ou non gouvernementales, des initiatives d'entreprises (comme celle des Eating cities $^{6}$ - http://www.eatingcity.org/) participent à l'approfondissement et à la diffusion de ce modèle.

Qu'elles promeuvent des eating cities innovantes ou des villes nourricières réconciliées avec la nature productive, ces initiatives proposent toutes des approches globales de l'alimentation en pensant de manière intégrée les acteurs de la production, de la transformation, de la distribution, mais aussi les pratiques de consommation et de recyclage. L'objectif étant de sortir des logiques de filières.

\section{Les enjeux d'une refondation métropolitaine de nos systèmes alimentaires}

La refondation de nos systèmes alimentaires urbains ${ }^{7}$ répond à un certain nombre d'enjeux : susciter une meilleure interaction entre espaces agricoles et espaces urbains ; garantir un cycle de vie des aliments optimisé; réduire l'empreinte écologique d'un marché globalisé ; ré-ancrer l'alimentation dans le territoire ; favoriser un cycle de vie optimisé par les aliments (économie circulaire); réaffirmer les patrimoines alimentaires locaux.

Cette refondation se donne donc des objectifs clairs et hiérarchisés. Tout d'abord ceux de souveraineté, de justice et de sécurité alimentaires : augmenter l'autonomie alimentaire locale (mais sans chercher à tout prix l'autosuffisance $)^{8}$. Il s'agit donc de privilégier désormais ce qui est produit localement, sans renoncer au reste et de payer le juste prix. Il ne s'agit pas de fermer les frontières mais de permettre le développement local d'entreprises agro-alimentaires proches des citoyens et d'intégrer plus avant ces entreprises locales dans les circuits de distribution existants dans les lieux publics.

Objectifs aussi d'accessibilité et de démocratisation alimentaires : permettre un accès amélioré aux aliments sains, nourrissants et culturellement adaptés, notamment via des lieux de proximité et des actions contre l'insécurité alimentaire ; favoriser une accessibilité physique, sociale, culturelle, financière ; repenser la répartition des différentes activités et infrastructures alimentaires dans le territoire métropolitain ; penser la nourriture comme un bien commun et pas seulement comme une marchandise; élaborer un nouveau modèle participatif de développement alimentaire.

Enfin, ce système alimentaire vertueux attendu cherche à encourager la diversité et l'économie 
alimentaires : en répondant à la demande des urbains pour une alimentation plus saine et en lien avec leur territoire (tout en prenant aussi en compte la diversité des cultures alimentaires) ; en se souciant des pratiques de consommation mais aussi en favorisant les nouveaux débouchés aux professionnels de la filière; en encourageant le développement d'entreprises responsables tant au niveau de la production, de la transformation, de la consommation que du recyclage alimentaire mais aussi en intégrant plus avant les entreprises agro-alimentaires régionales existantes dans les circuits de distribution existants.

\section{La gouvernance des villes en transition alimen- taire}

Pour arriver à ces objectifs, les villes développent un nouvel ensemble de coopérations entre différents acteurs dont l'enjeu alimentaire est « l'arène commune ». L'alimentation durable pour tous induit une gouvernance territoriale et transversale de l'alimentation comme clef de développement territorial; il s'agit d'impliquer l'ensemble des acteurs, notamment au sein d'instances de concertation et d'inventer de nouvelles modalités d'action fondées à la fois sur des politiques publiques et des initiatives locales visant une meilleure intégration des liens métropole/agriculture/alimentation. La métropole qui mange s'appuie donc à la fois sur des initiatives citoyennes, marchandes et publiques.

Les politiques publiques, que met en œuvre cette gouvernance alimentaire, visent à élaborer des stratégies susceptibles de contribuer au développement des systèmes alimentaires durables. Elles se présentent comme un programme et un cadre général d'objectifs et d'actions qui peuvent prendre des formes variables, plus ou moins abouties selon les métropoles. Certaines villes sont citées pour l'ambition et l'exhaustivité de ces programmes - comme Londres ou New York - ou encore pour leur créativité - comme Toronto, Montréal ou Québec. Chacune propose une approche éco-systémique de l'alimentation, en pensant de manière circulaire la chaîne des acteurs et les actions à mener.

La London Food Strategy prend ainsi en compte l'ensemble du champ alimentaire en construisant sa gouvernance autour de six axes, déployés par des organismes consultatifs ou opérationnels dédiés, à savoir: la vitalité commerciale ; la sécurité et l'engagement du consommateur ; le développement territorial : la santé dans les écoles; la réduction du gaspillage alimentaire et le recyclage des déchets. D'autres villes ébauchent à peine des stratégies centrées sur certaines étapes de l'approche globale, l'approvisionnement notamment.

Cette gouvernance s'appuie sur différents outils comme les labels (ainsi celui du réseau Délices - http://delice-network.org/), les réseaux (tel celui de l'IUFN, the international urban food network - http://www.iufn.org/en/), des think tanks ou des observatoires. Dans la rubrique des labels on peut aussi évoquer celui, emblématique, de l'UNESCO dédié à la gastronomie. En France se dessinent des projets portés notamment par des collectifs hybrides. Il en est ainsi pour Nantes Ville comestible (http:// www.nantesvillecomestible.org/) : un collectif pluridisciplinaire pour l'agriculture urbaine qui travaille à valoriser les réseaux et les acteurs en 
présence, à créer des alliances dans l'objectif de développer les pratiques de production alimentaires sur Nantes Métropole.

\section{Des marques publiques}

La gouvernance des villes en transition alimentaire, ce sont aussi des démarches centrées sur la qualité et la promotion des produits de terroir portées par des marques. Ainsi la marque CERVIA, la marque des produits alimentaires franciliens regroupe à la fois les missions d'un comité de promotion des produits agricoles et alimentaires franciliens et celle d'un centre régional d'innovation et de transfert (des produits et des savoir-faire agricoles et alimentaires franciliens). Elle soutient la pérennisation des entreprises alimentaires sur le territoire et construit un projet régional partagé pour l'ensemble des acteurs de la sphère alimentaire « du champ à l'assiette ». Cette marque ombrelle a déployé récemment la marque Saveurs Île-deFrance pour donner une visibilité et labelliser des produits issus de l'artisanat du territoire (http:// www.saveursparisidf.com/).

\section{De nouveaux intermédiaires}

La mutation de nos systèmes alimentaires passe aussi par des associations qui participent à la vulgarisation de l'agriculture urbaine, à sa mise en débat et invitent aux expérimentations : ainsi parmi les plus visibles « Vergers urbains » (http:// villecomestible.org/). Au fil de leurs actions, des relations et des interactions sociales se nouent autour de l'agriculture urbaine; des questionnements sur la nutrition et l'aménagement de l'espace urbain s'approfondissent. On ne saurait trop rappeler la dimension éducative du Grand Paris qui mange : pour aboutir à un système alimentaire viable et permettre de nouveaux apprentissages alimentaires collectifs, il est nécessaire de produire et de donner accès à une grande quantité de savoirs et de données. Ce modèle de développement repose sur une forte dynamique communautaire.

\section{Dynamiques citoyennes et communautaires pour un autre modèle}

Ainsi, nombre d'habitants s'engagent progressivement dans des actions de transformations urbaines, s'inventant jardiniers, botanistes, maraîchers, agriculteurs urbains. Mais paradoxalement, le métropolitain mange de moins en moins chez lui: il mange soit sur son lieu de travail, soit dehors, pour le meilleur ou pour le pire. Pourtant, les études montrent bien qu'une conscience alimentaire des effets de nos manières de manger sur notre environnement, à l'échelle de la planète mais aussi à celle notre santé, s'affirme dans les grandes villes. Par exemple, se dessine une citoyenneté alimentaire au fil de choix écologiquement viables et d'initiatives communautaires festives (repas de rue, banquet de quartier, pique-nique...) qui actualisent un souci de bien manger, sainement et en partage.

\section{Les lieux de la ville nourricière}

L'agriculture urbaine participe donc à la métropolisation (et au décentrement parisien), rassemblant autour d'une thématique forte des lieux innovants, les différents territoires et nouant des liens renouvelés entre Métropole/alimentation/agriculture. L'enjeu alimentaire devient un 
rendez-vous essentiel pour la gestion territoriale des territoires métropolitains. L'alimentation et plus avant l'agriculture urbaine (qui constituent des entrées pertinentes pour penser la cohésion métropolitaine) ne sont donc pas simplement envisageables en termes écologiques, environnementaux ou économiques mais aussi en termes culturels et patrimoniaux. Le développement métropolitain de l'AU représente une priorité en termes environnementaux et sociaux mais aussi économiques : il s'agit tant de promouvoir une agriculture locale de qualité, de réduire l'empreinte environnementale tout en renforçant l'autonomie alimentaire du territoire et en créant de l'emploi que de cimenter une collectivité autour de l'alimentation.

Les formes de l'AU sont très variées. Leur étude suppose une approche transversale en capacité de relier les initiatives à différentes échelles $-\mathrm{du}$ logement où l'on fait pousser des plantes aromatiques au parc agricole - et de penser ensemble initiatives amateurs et professionnelles. L'agriculture urbaine veut répondre aux défis alimentaires en repensant le territoire comme productif via une diversité d'outils : planification, protection mais aussi via un urbanisme alimentaire repensé et notamment un aménagement réfléchi qui valorisent les espaces productifs.

\section{Toits végétalisés 'productifs'}

Pour ce qui est de Paris, la capitale compte $40000 \mathrm{~m}^{2}$ de toitures végétalisées sur les équipements publics, 300 ruches dont 134 dans le domaine municipal, et a lancé en mai puis en novembre de nouveaux projets de végétalisation innovante dans l'espace public. L'objectif est d'ici 2020 d'aménager trente hectares de toitures et de façades pour la plantation de plantes et, à hauteur d'un tiers, de légumes et d'arbres fruitiers - et ceci dans une perspective pédagogique et collaborative.

\section{Espaces publics 'fertiles'}

L'agriculture urbaine réinvestit aussi des friches, des franges urbaines intégrant les principes d'un urbanisme alimentaire. Occupant une diversité croissante d'espaces disponibles, elle se voit attribuer des fonctions certes alimentaires mais aussi et peut-être surtout paysagères, sociales, pédagogiques, récréatives et environnementales. Derrière les objectifs de « préservation de la biodiversité » et d' " optimisation du cycle de vie des aliments », il s'agit aussi de repenser le rapport de l'alimentation aux lieux urbains, de ré-ancrer l'alimentation dans le territoire vécu. Sortir en grande partie des systèmes alimentaires dispersés mais sans se fermer au monde, aux produits venus d'ailleurs.

\section{Jardins comestibles 'à partager'}

Les jardins collectifs, partagés, communautaires sont autant de figures d'une pratique agricole qui naturalise la ville et restent la forme d'agriculture urbaine la plus courante. Souvent mis en place par des mouvements citoyens ou associatifs, ils permettent aussi des formes de sociabilité et d'apprentissage (qui construisent des capacités d'action dans et par la ville). Qu'ils soient privés, publics, communautaires, ils relèvent d'une micro agriculture occupant de petites surfaces et visant à produire sans impacter l'environnement. Leur fonction est donc tout à la 
fois environnementale, écologique et paysagère mais aussi récréative, sociale et culturelle, voire parfois économique. Ainsi les jardins d'insertion dans ses différentes formes permettent certes à chacun de mieux connaître le patrimoine végétal mais aussi de réintégrer la société via l'activité de production alimentaire dans une perspective tant sociale qu'économique ${ }^{9}$.

\section{Potagers marchands et bien commun}

Les acteurs du marché investissent donc les questions de l'agriculture urbaine pour les intégrer à leurs logiques économiques mais avec le soutien des acteurs publics. Acteurs publics et acteurs privés scellent ainsi des alliances assez inédites autour de l'alimentation de proximité. Ainsi Yannick Alléno a implanté avec le soutien de la Mairie de Paris un potager privatif (sur 3 étages), au dessus de son restaurant à la Mutualité, le Terroir parisien (http://www. yannick-alleno.com/carnet/terroir-parisien/). Ce potager est énoncé comme un lieu d'inspiration et d'expérimentation dédié à la table, soucieux de respecter la biodiversité en ville et de soutenir une production agricole en circuits courts pérennes. Le restaurateur cherche ainsi à relancer des variétés franciliennes oubliées (tel le radis violet de Gournay, le poireau de Viroflay, la courgette d'Orgeval) et à remettre au goût du jour des recettes franciliennes oubliées ${ }^{10}$.

\section{Fermes urbaines d'ici et d'ailleurs : entre ordi- naire et spectaculaire}

Elles sont nées aux États-Unis et au Canada fin des années 90 puis se sont développées en Amérique du Sud (citons l'expérience emblématique de Rosario où les fermiers urbains luttent contre la pauvreté dans le respect de l'environnement). Les fermes urbaines, qui permettent le développement local d'entreprises agroalimentaires d'un nouveau type (favorisant un accès amélioré aux aliments sains, une accessibilité à la fois géographique, sociale, financière et culturelle) participent à l'aménagement comestible des villes et à l'éducation alimentaire, bouleversant ainsi les codes traditionnels qui associent l'agriculture à la campagne et les villes aux activités industrielles de commerce et de services.

Parmi les expériences exemplaires à travers le monde, suscitant commentaires et médiatisations, citons aussi Brooklyn Grange, un projet aux enjeux sociaux et citoyens marqués ou encore les projets de Chicago (http://www.cityfarmchicago.org/), les Fresh City farms de Toronto (https://www. freshcityfarms.com/), celles de Vancouver (http:// innercityfarms.com/). L'on peut aussi évoquer Singapour et Sky Greens (www.skygreens.com), ces fermes verticales qui permettent de produire sur une surface au sol restreinte et en quantités significatives. Les projets de fermes verticales qui peuvent donner lieu à des gratte-ciel consacrés en grande partie à l'agriculture ${ }^{11}$ constituent des gestes architecturaux de grande ampleur, à forte dimension visuelle, créant fascination et bouleversant tant nos représentations de l'agriculture que celles du métier d'agriculteur. On voit ainsi depuis peu se démultiplier dans les médias spécialisés mais aussi généralistes de belles images visionnaires qui permettent de projeter une vision idéale, dotée d'une dimension universelle et annonçant un avenir inéluctable mais sans doute quelque peu dépolitisée. 
De plus en plus de villes se saisissent de l'agriculture verticale pour inventer de nouvelles formes urbaines. Et l'on peut se demander si l'avenir du Grand Paris passera par des fermes urbaines verticales. Ainsi les projets de l'architecte Vincent Callebaut (en réponse notamment à un appel à projet de la ville de Paris - « Smart City 2050 ») participent d'une critique du Paris énergivore et visent à résoudre les questions d'approvisionnement alimentaire à 1'horizon 2050. L'architecte questionne le métabolisme de la ville pour construire de nouvelles approches du système alimentaire de demain (http://vincent.callebaut.org/). Mais si le projet de Vincent Callebaut est très argumenté scientifiquement, on voit se démultiplier des visions alimentairement désincarnées de la ville autosuffisante, susceptibles d'intégrer des intérêts divers. Au-delà de l'imposition symbolique d'une certaine vision de la métropole de demain, on peut questionner la tentation de la biorégion et de l'autarcie agri-urbaine que celle-ci semble induire.

\section{Les fermes urbaines du Grand Paris}

Pour revenir au Grand Paris d'aujourd'hui, nous pouvons évoquer des projets plus confidentiels et dont la diversité dit bien la nécessité de ne pas s'arrêter à une seule définition des fermes urbaines. 'Ville fertile' est un projet collaboratif (http://www.vilefertile.paris/), une ferme urbaine participative, constituée en association maraîchère qui loue à la ville de Paris un terrain de $6000 \mathrm{~m}^{2}$ dans le bois de Vincennes (au jardin de l'Agronomie tropicale René Dumont). Elle se revendique de l'économie solidaire. Le projet Rurban ${ }^{12}$ à Colombes (www.r-urban.net), à la fois ferme, unité de recyclage et habitat écologique, associe des architectes, des économistes et des urbanistes, des sociologues mais aussi des artistes, des activistes, venant de toute l'Europe : cette initiative réalisée par l'Atelier d'Architecture Autogéré (l'architecte Constantin Petcou en est le fondateur) est une expérimentation qui pose notamment la question de la maîtrise foncière des espaces agricoles en ville La Recyclerie (http:// www.larecyclerie.com/) est un lieu hybride dans le XVIII ${ }^{\mathrm{e}}$ arrondissement, installée dans une gare, tout à la fois café, cantine, atelier et ferme urbaine, prairie et jardin aromatique qui se présente comme un terrain d'expérimentation écologique, un tiers lieu qui fait la part belle au low tech, au manuel, à la transmission et à la réappropriation des traditions utiles. On pourrait aussi citer en périphéries les fermes urbaines : Planète Lilas (http://planete-lilas.ouvaton.org/); la ferme expérimentale de Grignon (portée par Agroparistech), les Fermes en ville de Saint-Cyrl'École créée en 2014. Autant d'initiatives qui se font écho en ce qu'elles contestent les modèles de développement dominants et s'interrogent sur les modalités possibles et potentielles d'une transition vers des régimes plus durables : transition des systèmes alimentaires, transition des systèmes urbains. Ces fermes fournissent aussi bien les restaurants (de plus en plus en recherche de produits frais et sains provenant de productions locales) que les lieux publics.

\section{Circuits courts et culture lovavore}

Ces potagers et ces fermes alimentent des circuits courts reliant les producteurs aux consommateurs. Ces circuits se diversifient : aux AMAP s'ajoutent les marchés locaux, les cueillettes sur 
place, le troc aux plantes, les magasins de producteurs, les magasins intégrés, les tables d'hôte... mais aussi progressivement la grande distribution $^{13}$. Sans oublier l'importance croissante que prennent les médiations numériques permettant toutes sortes de livraisons à domicile.

L'agriculture semble ainsi investir tant et si bien la ville qu'on commence à parler d'urbanité agricole ou d'agrarisation de la ville. Progressivement, nous sommes sortis de l'approche en production de la ville comestible et nourricière pour la penser en termes de distribution et de consommation, d'usages et d'expériences culturelles et patrimoniales. Les habitants métropolitains sont globalement déconnectés du monde agricole, surtout en Île-de-France où aux petites fermes d'autrefois a succédé l'immensité des champs de blé, des paysages de grande culture, peu appréciés des citadins d'aujourd'hui qui n'ont tout simplement pas appris à les connaître. Avec l'agriculture urbaine se renoue le lien avec la terre : fermes, jardins et potagers urbains invitent aux activités agri-urbaines ; semer, planter, arroser, cueillir deviennent de nouveaux rituels urbains. L'intégration de l'agriculture urbaine dans la ville passe donc par la reconnaissance d'une possible urbanité agricole, c'est-à-dire d'une manière de vivre la ville et la métropole autrement.

\section{Transition}

L'alimentation semble ainsi pouvoir transformer non seulement les paysages urbains mais aussi les structures politiques, les espaces publics ou encore les liens sociaux et économiques qui les traduisent. Le projet Grand Paris 'nourricier' par- ticipe d'un nouveau modèle de développement, soucieux de la protection de la nature - terme que nous privilégierons sur ceux, plus techniques, de biosphère et de biodiversité, postulant une transformation de soi, de sa manière d'habiter, de consommer, de partager, de mettre en commun. Il postule une évolution en profondeur de nos liens à la nature (de ménagement et non de domination) et à la conservation de la vie. Pour autant, il reste du chemin à parcourir pour une véritable intégration des acteurs, des activités et des espaces de l'agriculture et de l'alimentation urbaines.

Mais il n'est pas lieu ici de conclure car cet article n'est que la première partie d'un ensemble : les processus de patrimonialisation, d'évènementialisation, de mise en tourisme et donc de mises en récit de ce « Grand Paris qui mange » seront ainsi envisagés dans un prochain numéro de Quaderni. 


\section{$R \cdot \dot{E} \cdot F \cdot \dot{E} \cdot R \cdot E \cdot N \cdot C \cdot E \cdot S \quad N \cdot O \quad \cdot \quad T \cdot E \cdot S$}

BRAND (Caroline) et BONNEFOY (Serge), 1. Ces textes sont issus d'une communication et «L'alimentation des sociétés urbaines : une cure d'une journée d'étude organisée par l'auteur en mai de jouvence pour l'agriculture des territoires et novembre 2015 à l'ISCC puis à l'Université de la métropolitains », in Vertigo - la revue électro- Sorbonne.

nique en sciences de l'environnement, Volume 11, numéro 2, septembre 2011.

KRAUSZ (Nicolas), LACOURT (Isabelle), MARIANI (Maurizio), La ville qui mange, Éditions Charles Léopold Mayer, 2013.

TORRE (André) et BOURDEAU-LEPAGE (Lise), Quand l'agriculture s'installe en ville, http://www.metropolitiques.eu/Quand-l-agriculture-s-installe-en.html, 2014.

VIVREENVILLE.ORG, Les villes nourricières, mettre l'alimentation au cour des collectivités, coll. « Outiller le Québec », Vivre en Ville éditions, 2014.

2. En 2012, les repas distribués dans la restauration collective (plus de 30 millions) comptaient $20 \%$ d'aliments dits durables. Les projets d'approvisionnement local pour la restauration collective sont souvent mobilisés pour illustrer l'intervention des villes (et plus largement des collectivités territoriales), pour témoigner de leur volonté de construire de nouvelles légitimités et amorcer de nouveaux liens avec les acteurs agricoles.

3. Au niveau national, signalons que l'année 2014 a aussi été marquée par le lancement du Pacte national de Lutte contre le gaspillage alimentaire et la Loi d'« avenir pour l'agriculture, l'alimentation et la forêt » (intégrant la dimension « projet alimentaire territorial »).

4. Voir l'exposition à la Cité de l'Architecture et du patrimoine, La Ville Fertile : http://www.citechaillot. fr/fr/expositions/expositions_en_location/24047la_ville fertile.html

5. Villes Nourricières : https://vivreenville.org/villesnourricieres. La ville qui mange $:$ http://www.eclm.fr/ ouvrage-361.html

6. Un programme lancé en 2010 par le consortium italien RISTECO - Terre Citoyenne - co-fondé par la fondation Léopold Meyer pour le Progrès de l'homme. En fait, ce consortium est lié à une entreprise SOTRAL - SPA spécialisée dans les services de logistique et du transport alimentaire à destination de la restauration publique.

7. Un système alimentaire durable est un réseau de collaboration qui intègre la production et la consommation de productions alimentaires et la gestion des 
matières résiduelles, dans le but d'accroître la santé environnementale, économique et sociale de la ville.

8. Pour rappel, l'Île-de-France consomme un million de tonnes de fruits et légumes par an mais elle n'en produit que $15 \%$, la moitié des exploitations maraîchères ayant disparu, principalement à cause de l'urbanisation.

9. Voir le programme JASSUR (jardins associatifs urbains et villes durables) et le réseau des jardins partagés en France (www.jardins-partagés.org).

10. On peut aussi citer le Potager de l'hôtel Pullman pour lequel le «nourricier» devient un attribut de la renommée : http://topager.com/portfolio-item/jardinpotager-restaurant-ou-hotel/.

11. L'idée de base de ces projets vient d'un concept développé en 1999 par Dikson Despommier, professeur en santé environnementale et microbiologie à l'université de Columbia à New York en association avec des étudiants diplômés de la Medical ecology class.

12. Un projet de circuits courts qui regroupe trois dispositifs : Recyclos, Agrocité et Ecohabitat.

13. Rappelons l'importance de la question des circuits courts dans le plan alimentation durable pour Paris 2015-2020 qui a été adopté le $1^{\text {er }}$ juillet.

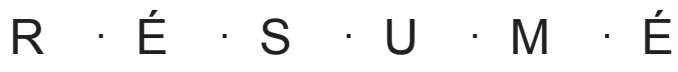

Les grandes métropoles sont confrontées à la question alimentaire de multiples manières : en termes d'approvisionnement, de sécurité (les crises à répétition) et de souveraineté, mais surtout en termes de gouvernance. Les "métropoles nourricières ", dont les promesses se démocratisent et se médiatisent avec une grande inventivité, deviennent un enjeu tant politique, culturel que symbolique. Pourquoi et comment adapter culturellement notre espace, nos systèmes, nos comportements pour qu'ils prennent pleinement en compte la nécessaire transition alimentaire ? Cet article (le premier d'une série) se centre sur le "Grand Paris qui mange », comme échelle pertinente pour déployer des projets et des politiques tant en termes d'agriculture urbaine que d'actions autour des cultures alimentaires, culinaires et gastronomiques renouvelées.

\begin{abstract}
Contemporary metropolises are facing important questions surrounding food in terms of supply, security (the repeated crises) and sovereignty, but especially in terms of governance. The "eeder cities", whose promises are democratizing and mediatized with great inventiveness, become a political issue, at once cultural and symbolic. Why and how to culturally adapt our space, our systems, our behavior so that they take full account of necessary food transitions?
\end{abstract}


